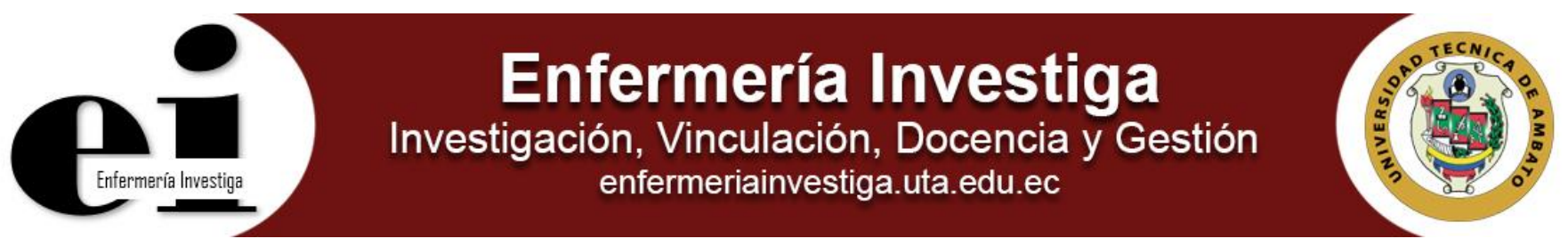

Artículo original

\title{
Diagnóstico situacional de la Carrera de Enfermería
}

Situational diagnosis of the Nursing Career

Diana Nancy Martínez García1, Eulalia Isabel Analuisa Jiménez¹, José Luis López Herrera ${ }^{1}$, Alexandra Laguapillo Vergara' ${ }^{1}$, Miguel Ángel de la Fuente Briz ${ }^{1}$, Carmen de las Mercedes Cevallos Méndez ${ }^{1}$, Miriam Ivonne Fernández Nieto ${ }^{1}$, Graciela de las Mercedes Quishpe Jara ${ }^{1}$

\footnotetext{
${ }^{1}$ Universidad Técnica de Ambato - Facultad de Ciencias de la Salud - Carrera de Enfermería - Ambato - Ecuador.
}

Martínez GDN, et al. Diagnóstico situacional de la Carrera de Enfermería. Enferm Inv (Ambato). 2017; 2(3):104-109.

2477-9172 / 2550-6692 Derechos Reservados @ 2017 Universidad Técnica de Ambato, Carrera de Enfermería. Este es un artículo de acceso abierto distribuido bajo los términos de la Licencia Creative Commons, que permite uso ilimitado, distribución y reproducción en cualquier medio, siempre que la obra original es debidamente citada.

\section{Historia:}

Recibido: 02 agosto 2017

Revisado: 10 agosto 2017

Aceptado: 14 agosto 2017

Palabras Claves: Enfermería; educación en salud; investigación

Keywords: Nursing; health education; research

\section{Resumen}

Introducción: La Carrera de Enfermería responde a las necesidades en el entorno de salud identificadas en la zona central del país. Está debidamente planificada acorde a las instancias científicas y técnicas del momento.

Objetivo: Diagnosticar la situación actual de la Carrera de Enfermería de la UTA en función de las necesidades del entorno en el cual se desarrolla y el encargo social de la misma.

Métodos: Se realizó un estudio observacional, descriptivo y retrospectivo. El periodo de estudio abarcó de abril a septiembre del año 2015, el universo de estudio estuvo integrado por los 46 docentes que laboraban en la carrera en ese periodo y no se seleccionó muestra.

Resultados: La planta docente solo contó con dos docentes titulares auxiliares, tan solo el $31.8 \%$ eran docentes a tiempo completo. El 95\% de los docentes tuvo afinidad con las cátedras que imparten. Las actividades de vinculación con la colectividad y la gestión mayormente recayeron en los docentes a tiempo completo. Durante el periodo fueron publicados cuatro libros y el $73 \%$ de los graduados se encontraron como empleados públicos.

Conclusiones: No se cuenta con programas de posgrado en aras de mejorar la afinidad de estos a la catedra que imparten los docentes. En el área de investigación no se ha potencializado la creación de grupos de investigación multidisciplinarios, que a la vez involucren a los estudiantes de pregrado, de manera que se fomente una dinámica de investigación desde que se inicia el estudiante en su profesión.

\section{Abstract}

Introduction: The Nursing Career responds to the needs in the health environment identified in the central area of the country. It is duly planned according to the scientific and technical instances of the moment.

Objetive: To diagnose the current situation of the Nursing Career of the UTA according to the needs of the environment in which it is developed and the social order of the same.

Methods: An observational, descriptive and retrospective study was performed. The study period covered from April to September of 2015, the universe of study was composed of 46 teachers who worked in the race in that period and was not selected sample.

Results: The teaching staff only had two auxiliary teachers, only $31.8 \%$ were full-time teachers. $95 \%$ of teachers had affinity with the chairs they teach. The activities of association with the community and management mainly fell to full-time teachers. During the period four books were published and $73 \%$ of the graduates found themselves as public employees.

Conclusions: There are no postgraduate programs in order to improve their affinity with the professorships taught by teachers. In the area of research, the creation of multidisciplinary research groups has not been strengthened, while involving undergraduate students, in order to foster a research dynamic since the student starts in his profession. 


\section{Introducción}

Alineada a los objetivos del Plan Nacional del Buen Vivir, la Agenda Zonal 3 del Ecuador y enmarcada en la zona de impacto de la Universidad Técnica de Ambato (UTA), la Carrera de Enfermería tiene la responsabilidad de formar profesionales en la región central del país para satisfacer las demandas del entorno en el cual se desarrolla. Es por ello, por lo que se debe tributar al desarrollo sociocultural, económico, científico-técnico y ambiental de los habitantes de la zona1. Para lograr tales objetivos se propone un programa de pregrado que culmina con la titulación de tercer nivel denominada "Licenciado en Enfermería", lograda por medio de procesos innovadores y con una visión prospectiva que contribuya positivamente a la solvencia de los problemas de salud identificados en la zona².

La Carrera de Enfermería de la UTA fue creada el 5 de agosto de 1997 según la resolución de Consejo Universitario N. 529-97 CU-P y con una duración de estudios de nueve semestres incluyendo el trabajo de titulación. Esta responde a las necesidades en el entorno de salud identificadas en la zona central del país. La carrera está debidamente planificada acorde a las instancias científicas y técnicas del momento ${ }^{1}$. Cabe destacar que, dentro del proceso de aprendizaje, se implementan metodologías prácticas que aseguran la excelencia académica y la labor del profesional en las actividades inherentes a su campo de acción; toda vez que se dispone de los recursos materiales y humanos necesarios para ${ }^{3}$.

Esta labor de carácter social y humanista persigue mejorar la calidad de vida de la población por medio de la intervención no solo a nivel del individuo, sino también a nivel de la familia y la comunidad mediante el trabajo en equipo multidisciplinario. En tal sentido ese accionar se ha enfocado hacia nuevas líneas en la atención a enfermedades crónicas no trasmisibles, que constituyen las primeras causas de morbilidad y mortalidad en la actualidad, tal es el caso de las enfermedades cardiovasculares, respiratorias crónicas, la atención a la paciente materna, atención al niño, consumo de drogas de abuso y enfermedades infecciosas de alta prevalencia como la Tuberculosis ${ }^{4,5}$.

El objetivo del presente artículo es diagnosticar la situación actual de la Carrera de Enfermería de la UTA en función de las necesidades del entorno en el cual se desarrolla y el encargo social de la misma, por medio de sus pilares academia, investigación, vinculación con la colectividad y gestión; acorde a los objetivos del Plan Nacional del Buen Vivir y la Agenda Zonal 3, para contribuir a la transformación de la matriz productiva.

\section{Materiales y métodos}

Se realizó un estudio observacional, descriptivo y retrospectivo con el propósito de diagnosticar la situación actual de la Carrera de Enfermería de la UTA. El periodo de estudio abarcó de abril a septiembre del año 2015, el universo de estudio estuvo integrado por los 46 docentes que laboraban en la carrera en ese periodo y no se seleccionó muestra. Como fuente de información secundaria fueron utilizados los distributivos de trabajo del personal docente y los registros del Sistema Institucional de Seguimiento a Graduados durante el periodo antes señalado. Se confeccionó una base de datos automatizada en el software SPSS 22.0 y el análisis estadístico se realizó a través de medidas descriptivas de frecuencia relativa y absoluta en correspondencia con el tipo de variable estudiada. El diagnóstico interno y externo situacional de la carrera, se hizo por medio de la matriz FODA desarrollada por la totalidad de la planta docente.

\section{Resultados}

La Carrera de Enfermería está debidamente planificada acorde a las instancias científicas y técnicas del momento. Cabe destacar que dentro del proceso de aprendizaje se implementan metodologías prácticas que aseguran la excelencia académica y la labor del profesional en las actividades inherentes a su campo de acción. La carrera dispone de recursos necesarios para ello y cuenta con la suficiente experiencia que garantiza la programación establecida.

\section{Academia}

La Carrera de Enfermería contó con 44 docentes, de los cuales solo dos presentan categoría de titular auxiliar, y el resto de docentes son ocasionales o no titulares. En cuanto a la dedicación de los docentes, el 31.8\% corresponde a docentes a tiempo completo, al tiempo que el $68.2 \%$ se encontró a medio tiempo o tiempo parcial. Relacionado con la afinidad del título de posgrado del docente con la asignatura que dicta, el 95\% de los docentes tuvo afinidad con las cátedras que imparten, siendo la meta llegar al 100\% para el desarrollo óptimo del proceso de enseñanza aprendizaje en la formación de los estudiantes.

En la figura 1 se puede observar que casi casi la mitad de los docentes de los docentes (43.2\%) se dedican a otras actividades referentes a la academia como es el caso de tutorías y prácticas preprofesionales; al tiempo que la gestión y la vinculación mayormente recae sobre los docentes a tiempo completo.

Figura 1. Distribución de actividades complementaria de docentes de la Carrera de Enfermería.

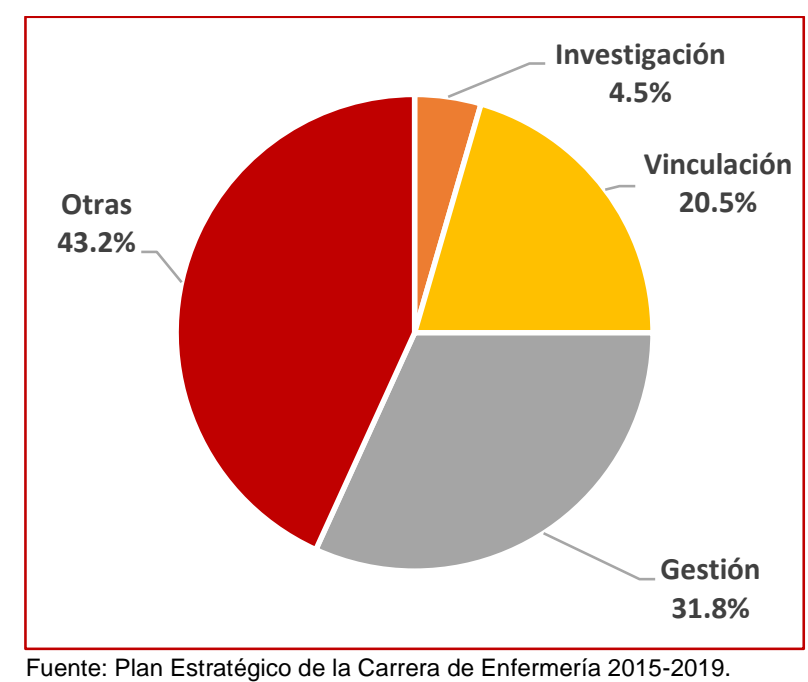

Las cifras estadísticas acerca de las postulaciones por carrera en el periodo marzo 2015, evidenciaron la demanda de la carrera. El número de postulados para el período marzo 2015 fue de 1307 para la Carrera de Enfermería, posicionándose como la segunda opción de postulación por el estudiante de nivel secundario. 


\section{Investigación}

La investigación en la carrera se gestiona y apoya eficazmente con criterios e instrumentos innovadores por parte de la Unidad Operativa de Investigación y Desarrollo de la Facultad de Ciencias de la Salud de la Universidad Técnica de Ambato, colaborando y potenciando esta área por medio de la transferencia de sus resultados, al servicio de la sociedad. Durante el periodo de estudio fueron publicados cuatro libros por parte de docentes de la carrera, los cuales aportan al microcurriculum y se incorporarán como bibliografía.

\section{Vinculación}

La Carrera de Enfermería se compromete con la participación en el proceso de creación social de la cultura y de la transformación de la comunidad. Actualmente y acorde a las necesidades que se identifican en la comunidad, se desarrollan nueve proyectos de vinculación; estos proyectos forman parte de un proceso dinámico interactivo de la carrera con su entorno para solventar las necesidades de la comunidad. Existe una disposición y participación de los sectores más necesitados, junto al apoyo de las autoridades, estudiantes y docentes como parte de la realidad de este proceso. Gradualmente se han incrementado los convenios con instituciones públicas para el despliegue de las actividades de vinculación con la colectividad.

Como parte del seguimiento a graduados de la carrera, se evidenció que el $73 \%$ de los graduados manifiesto ser empleado público, el $21 \%$ estuvo vinculado al sector privado y los empleados autónomos representaron un $6 \%$ del total de la muestra.

Tabla 1. FODA Carrera de Enfermería, fortalezas.

\begin{tabular}{|c|c|}
\hline Academia & Vinculación \\
\hline $\begin{array}{l}\text { - Plan de estudios pertinente a la formación de profesionales } \\
\text { acorde a la demanda social de la población. } \\
\text { - Aplicación de perspectiva de género en las asignaturas que } \\
\text { aplica. } \\
\text { - Docentes con suficiente experiencia profesional y académica. } \\
\text { - Elevado número de docentes con actualización científica } \\
\text { continua (98\%). } \\
\text { - Metodología múltiple de enseñanza que incluye laboratorios de } \\
\text { simulación. } \\
\text { - Planificación y ejecución de tutoría académica acorde a las } \\
\text { necesidades de aprendizaje de los alumnos. }\end{array}$ & $\begin{array}{l}\text { - Elevado porcentaje de estudiantes que participan en los } \\
\text { proyectos de vinculación. } \\
\text { - Aumento de proyectos de vinculación con impacto en la zona. } \\
\text { - Suficientes convenios con sectores estratégicos. } \\
\text { - Proyectos de vinculación de conjunto con la Red de Carreras de } \\
\text { Enfermería. } \\
\text { Seguimiento a graduados. } \\
\text { - Bolsa de Empleo. } \\
\text { - Oferta de superación continua y posgrado acorde a las demandas } \\
\text { identificadas en los graduados. } \\
\text { Prácticas preprofesionales. } \\
\text { - El alumno practicante se encuentra tutorado por personal de } \\
\text { alta experiencia de las Unidades Docentes Asistenciales (UADS) } \\
\text { que no pertenece a la carrera. } \\
\text { - Elevada capacitación de docentes asesores en la práctica } \\
\text { preprofesionales } \\
\text { - Participación del docente asesor en el desarrollo del Proceso de } \\
\text { Atención de Enfermería (PAE). }\end{array}$ \\
\hline Investigación & Gestión \\
\hline $\begin{array}{l}\text { - Se cuenta con los recursos tecnológicos y materiales que } \\
\text { garantiza el desarrollo investigativo de la carrera. } \\
\text { - Se cuenta con talento humano capacitado. } \\
\text { - Tres proyectos presentados en fase de aprobación. } \\
\text { - Amplia participación de la planta docente en actividades } \\
\text { científicas como congresos y seminarios a nivel nacional e } \\
\text { internacional. } \\
\text { - Participación de docentes en proyectos de investigación en } \\
\text { colaboración con otras Carreras o Facultades de la Universidad } \\
\text { Técnica de Ambato. } \\
\text { - Convenios nacionales e internacionales de cooperación } \\
\text { científica. } \\
\text { - Aprobación por la SENESCYT de una revista científica } \\
\text { desarrollada en la carrera. }\end{array}$ & $\begin{array}{l}\text { - Se cuenta con responsables en las áreas académicas de la } \\
\text { Carrera (Coordinadores de vinculación, UPE, investigación. } \\
\text { Seguimiento a graduados y prácticas pre profesionales). } \\
\text { - Locales de profesores con las facilidades necesarias, internet por } \\
\text { wifi y red, ordenadores y equipamiento de oficina. } \\
\text { - Áreas para el desarrollo de actividades docentes prácticas. }\end{array}$ \\
\hline
\end{tabular}

Fuente: Plan Estratégico de la Carrera de Enfermería 2015-2019.

Tabla 2. FODA Carrera de Enfermería, debilidades.

\begin{tabular}{|l|l|}
\hline \multicolumn{1}{|c|}{ Academia } & \multicolumn{1}{c|}{ Vinculación } \\
\hline $\begin{array}{l}\text { - Tasa actual de titulación en un 47\%. } \\
\text { - Déficit de convenios institucionales para la superación } \\
\text { profesional de docentes a nivel nacional e internacional. }\end{array}$ & $\begin{array}{l}\text { - Recursos materiales y económicos limitados. } \\
\text { - Escaso número de docentes con título de PhD. }\end{array}$ \\
$\begin{array}{l}\text { - Escasa docentes y estudiantes a diferentes sectores. } \\
\text { comunidad. }\end{array}$
\end{tabular}


- Limitado número de docentes con titularidad y tiempo completo.

- Fondo bibliográfico físico limitado.

- Carga excesiva en labores de gestión a los docentes.

- Limitada oferta de cátedras optativas.

- Limitado uso de nuevas tecnologías aplicadas a la gestión y práctica docente

- Desarticulación de la vinculación y la investigación.

Seguimiento a graduados.

- Número de horas limitadas.

- No existe capacitación a los responsables de forma permanente

Prácticas preprofesionales.

- Deficiente integración de docentes que realizan prácticas preprofesionales con docentes que imparten teoría.

- Deficiente sistema de incentivo, motivación y reconocimiento académico.

\section{Investigación}

- Insuficiente motivación de docentes para desarrollar investigación científica.

- Escaso número de investigadores.

- No inserción de la carrera en redes de desarrollo científico a nivel internacional.

- Falta de motivación de los docentes para realizar publicaciones de los estudios realizados.

- Escaso número de publicaciones indexadas en la carrera. Fuente: Plan Estratégico de la Carrera de Enfermería 2015-2019.

Plantearon los encuestados que reciben la información sobre las ITS en la siguiente prioridad (tabla 3), primero por los medios de difusión ( $n=48$ 80.0\%), segundo por los amigos ( $n=4575.0 \%$ ), con el $68.3 \%$ y $60.0 \%$ ubicaron a los profesores y padres respectivamente.

Tabla 3. FODA Carrera de Enfermería, oportunidades.

\begin{tabular}{|c|c|}
\hline Academia & Vinculación \\
\hline $\begin{array}{l}\text { - Firma de convenios con otras IES. } \\
\text { - Proceso de acreditación institucional. } \\
\text { - Prestigio de la Universidad en Estudios Superiores a nivel de la } \\
\text { región central del país. }\end{array}$ & $\begin{array}{l}\text { - Interrelación de la UTA con sociedad local, regional y nacional. } \\
\text { - Interés de los medios de difusión pública para la difusión de los } \\
\text { - Convenios tanto en el sector público y/o privado } \\
\text { - Apertura de las instituciones de salud pública y privada para la } \\
\text { realización de los proyectos de vinculación. } \\
\text { Seguimiento a graduados. } \\
\text { - Sistema de Seguimiento a Graduados. } \\
\text { - Elevada demanda de graduados de la carrera por parte de } \\
\text { instituciones públicas y/o privadas. } \\
\text { Prácticas preprofesionales. } \\
\text { - Suscripción de convenios con instituciones de salud públicas o } \\
\text { privadas. } \\
\text { recursos materiales que garanticen el desarrollo adecuado de las } \\
\text { prácticas pre profesionales. }\end{array}$ \\
\hline Investigación & Gestión \\
\hline $\begin{array}{l}\text { - Existencia de redes de desarrollo científico a nivel nacional e } \\
\text { internacional. } \\
\text { - Suscripciones de la universidad para la capacitación de docentes } \\
\text { en temas de investigación. } \\
\text { - Producción científica que se genere a partir de proyectos en } \\
\text { desarrollo. } \\
\text { - Existencia de un elevado número de revistas indexadas en base } \\
\text { de datos regionales (LATINDEX) y de alto impacto como (SCOPUS } \\
\text { e ISI WEB) afines a la Carrera de Enfermería. }\end{array}$ & $\begin{array}{l}\text { - Apoyo de las autoridades de la Universidad. } \\
\text { - Políticas que auspician la capacitación docente a nivel nacional e } \\
\text { internacional. } \\
\text { - Disponibilidad de un Plan Estratégico Institucional. } \\
\text { - Disponibilidad de un Plan de Mejoras institucional y de carrera. } \\
\text { - Disposición por parte del CES para la aprobación de estudios de } \\
\text { postgrado de la Carrera de Enfermería. } \\
\text { - Asociación Ecuatoriana de Facultades de Enfermería (ASEDEFE). }\end{array}$ \\
\hline
\end{tabular}

Fuente: Plan Estratégico de la Carrera de Enfermería 2015-2019.

Tabla 4. FODA Carrera de Enfermería, amenazas.

\begin{tabular}{|l|l|}
\hline \multicolumn{1}{|c|}{ Academia } & \multicolumn{1}{c|}{ Vinculación } \\
\hline $\begin{array}{l}\text { - Remuneración económica superior en instituciones de salud } \\
\text { públicas. }\end{array}$ & $\begin{array}{c}\text { •Áreas destinadas a actividades de vinculación que no cuentan } \\
\text { con los recursos necesarios. }\end{array}$ \\
- Alto índice de migración de los estudiantes debido a la plaza \\
asignada de internado rotativo.
\end{tabular}




\begin{tabular}{|c|c|}
\hline $\begin{array}{l}\text { - Competencia por el mercado laboral y la presencia de otras } \\
\text { universidades en la zona centro del país. }\end{array}$ & $\begin{array}{l}\text { - Insuficiente aporte en recursos económicos y materiales por } \\
\text { parte de las organizaciones beneficiarias para la ejecución de los } \\
\text { proyectos. } \\
\text { Seguimiento a graduados. } \\
\text { - Falta de compromiso de los graduados en el desarrollo de la } \\
\text { carrera. } \\
\text { - Discontinuidad en las políticas institucionales. } \\
\text { Prácticas preprofesionales. } \\
\text { - Falta de interés en algunos responsables de docencia e } \\
\text { investigación de las UAD. }\end{array}$ \\
\hline Investigación & Gestión \\
\hline $\begin{array}{l}\text { - Mercado altamente competitivo en instituciones de Educación } \\
\text { Superior. } \\
\text { - Asignación de } 24 \text { horas de clases semanales a docentes tiempo } \\
\text { completo, según el Reglamento del Régimen Académico. }\end{array}$ & $\begin{array}{l}\text { - Reducción presupuestaria. } \\
\text { - Demoras en los mecanismos de aprobación de programas de } \\
\text { posgrado. }\end{array}$ \\
\hline
\end{tabular}

Fuente: Plan Estratégico de la Carrera de Enfermería 2015-2019.

\section{Discusión}

Como parte del proceso de acreditación de las carreras de enfermería a nivel nacional, han sido las actividades de gestión y vinculación con la colectividad, puntos clave en esta evaluación ${ }^{5}$. En tal sentido, la carrera ha ubicado en estas actividades a su planta docente a tiempo completo. Tanto las actividades de gestión, como las actividades de extensión universitaria a la comunidad demandan varias horas de trabajo; lo cual unido a la complejidad de su coordinación, hace que sea el personal a tiempo completo el más idóneo 6 (figura 1).

\section{Análisis interno}

Ante el bajo índice de titularidad que presenta la carrera, debe analizarse la asignación de puestos a docentes titulares, toda vez que el Consejo de Evaluación, Acreditación y Aseguramiento de la Calidad de la Educación Superior (CEAACES) establece como meta el 60\%, contra el $80 \%$ que exige el Consejo de Educación Superior (CES) ${ }^{5}$.

Según lo establecido por el CEAACES y el CES, la carrera debe optimizar la dedicación de los docentes, pues son los docentes a tiempo completo sobre los cuales recae la mayor responsabilidad de la academia y demás pilares; sin embargo, es oportuno resaltar que los docentes encargados de las prácticas pre profesionales en su totalidad laboran a medio tiempo en la carrera, pues cumplen contrato a tiempo completo en instituciones del Ministerio de Salud Pública (MSP) o del Instituto Ecuatoriano de la Seguridad Social $(\text { IESS) })^{2}$.

Durante el periodo estudiado la carrera contó con un docente Doctor en Ciencias ( $\mathrm{PhD}$, siglas en inglés) que impartió docencia afín con su título. En este sentido es necesario que la universidad cree las facilidades a los docentes con interés para la realización de este tipo de estudios, independientemente se trate de docentes titulares u ocasionales ${ }^{7}$.

Solo dos docentes estuvieron vinculados a la investigación, en este particular debe considerarse también a los docentes a medio tiempo que laboran en las instituciones de salud, pues su actividad asistencial puede verse reflejada a la investigación dentro de la carrera ${ }^{8}$.

La demanda de la carrera por los estudiantes del Sistema Nacional de Nivelación y Admisión (SNNA) se ha venido incrementado cada año, a razón de un $12 \%$ desde el año 2013 al año 2015.
Referente a la producción científica, debe lograrse la publicación de artículos científicos ya sea en bases de datos de alto impacto o regionales. En contraste a esta realidad, todos los docentes de la carrera han logrado presentaciones de ponencias en eventos científicos de carácter internacional, logro que debe mantenerse a mediano y largo plazo.

En cuanto a la vinculación con la colectividad y los proyectos que se han desarrollado, los insumos y logística necesaria pueden deben ser optimizados, así como equipamiento necesario, lo que repercutiría en un mayor alcance de los proyectos de vinculación. Por otro lado, las instituciones beneficiarias no realizaron un aporte suficiente para el desarrollo y ejecución de los proyectos, pues no lo consideraron de su responsabilidad.

\section{Análisis externo}

Se imponen a futuro cambios cualitativos en la Carrera de Enfermería de la UTA, acorde a los parámetros de los entes reguladores superiores. Tal es el caso de los criterios de admisión a la carrera, los cuales no solo deben circunscribirse al curriculum de la educación secundaria, sino que debe tenerse en cuanta el factor humano y orientación vocacional de los aspirantes para el ejercicio de la profesión ${ }^{5}$.

Deben ser incorporadas las nuevas metodologías de evaluación formativa y reducirse los componentes especializados $^{9,4}$. De esta forma se priorizan las metodologías más transversales, pero por encima de todo debe inculcarse a los estudiantes el hábito de aprendizaje a lo largo de la vida "autoaprendizaje" y el sentido crítico "autoevaluación"; con la introducción de las metodologías educativas activas que fomentan el aprendizaje más allá de la actividad docente convencional ${ }^{9}$.

El desarrollo tecnológico de las últimas décadas ha situado al profesional de la enfermería frente a un escenario nuevo. Se debe tomar consciencia que, la tecnología no solo abrirá revolucionarias formas de diagnóstico o novedades terapéuticas, sino también nuevas relaciones tanto entre el paciente y el enfermero ${ }^{9}$. De acá se desprende la necesidad de incorporar en el currículo el colectivismo, así como desarrollar aprendizajes con el uso de las Tecnologías de Información y Comunicación (TICs), la interconexión, la telemedicina y el trabajo en redes ${ }^{5}$. 


\section{Campos que provee implementar a futuro.}

En los modelos contemporáneos y enfoques sistémicos del conocimiento, se describe el "Meta paradigma en Enfermería", desarrollándose con un componente de mayor abstracción teórica. Este se apoya en las teorías para su aplicación en la práctica, definiéndose cuatro constructos: persona, salud, sociedad-ambiente y cuidado de enfermería ${ }^{7,8}$. El desarrollo actual de la disciplina incluye a los modelos conceptuales que permiten, junto a las teorías, definir una estructura y lenguaje comunes; con diferentes niveles de aplicabilidad en la práctica desde la descripción y comprensión del fenómeno, hasta la interpretación, la explicación y la predicción.

\section{Conclusiones}

Finalmente, es necesario consolidar una estructura académica sólida en la universidad, sobre la base de la actualización de sus procedimientos. No se cuentan con programas de posgrados en la universidad que direccionen a la afinidad entre la catedra y la formación de posgrado de la planta docente, toda vez que el desarrollo de estos consolidaría la educación continua y virtual. En el área de investigación no se ha potencializado la creación de grupos de investigación multidisciplinarios, que a la vez involucren a los estudiantes de pregrado, me manera que se fomente una dinámica de investigación desde que se inicia el estudiante en su profesión.

\section{Conflicto de intereses}

Ninguno declarado por los autores.

\section{Referencias}

1. Senplades. Inicio - Plan Nacional 2013 - 2017 [en línea]. 2013 [citado 17 de septiembre de 2017]. Disponible en: http://www.buenvivir.gob.ec/

2. UTA Plan de Desarrollo Institucional Universidad Técnica de Ambato [en línea]. 2015 [citado 17 de julio de 2017]. Disponible en: https://www.uta.edu.ec/v3.0/pdf/planes/planestrategico2015-2019-1612-2016.pdf

3. Moreno G, Martínez D, Fernández M, Quishpe $G$ de las $M$. Seguimiento a graduados de la Carrera de Enfermería de la Universidad Técnica de Ambato. Enfermería Investiga: Investigación, Vinculación, Docencia y Gestión [en línea] 27 de marzo de 2017 [citado 17 de junio de 2017];2(1, Mar):23-7. Disponible en: https://enfermeriainvestiga.uta.edu.ec/index.php/enfermeria/article/vi ew/46

4. 4. Matheus M, Mendoza de Graterol E, González de Uzcategui A, Villalobos E, Mendoza G, Fernández SC, et al. Evaluación curricular y diagnóstico situacional de la Escuela de Enfermería. [citado 2 de junio de 2017]; Disponible en http://www.ucv.ve/fileadmin/user upload/vrac/documentos/Curricular Documentos/Evento/Ponencias 3/matheus milagros y otros.pdf

5. Herrera LAB, Gonzales EMF, Jaramillo NDG, Simbaña RMJ, Ulloa MNO. La Educación Superior y el Proceso de Transformación Social en el Ecuador. Quipukamayoc [en línea]. 2014 [citado 11 de juio de 2017];22:187-200. Disponible en: http://revistasinvestigacion.unmsm.edu.pe/index.php/quipu/article/vie w/11061

6. Montoya MSR, Peñalvo FJG. Movimiento educativo abierto. Virtualis [en línea]. 2016 [citado 11 de julio de 2017];6(12):1-13. Disponible en: http://aplicaciones.ccm.itesm.mx/virtualis/index.php/virtualis/article/vi ew/125

7. Alfonso P, Alejandra M, Navas Salcedo AT, et al. Desarrollo Histórico del Programa de Enfermería de la Universidad Cooperativa de Colombia, Sede Bucaramanga, en el período 1994 a 2014: Perspectiva de los egresados. [en línea] [B.S. thesis]. 2017 [citado 10 de julio de 2017]; Disponible en http://repository.ucc.edu.co/handle/ucc/281

8. Viltres MP, Viltres KL, Puebla ER, Olivera DLH, Verdecia NO. Formación de recursos humanos de Enfermería y personal docente. Un reto hecho realidad. Yara 2015. MULTIMED Revista Médica Granma [Internet]. 2016 [citado 9 de junio de 2017];20:557-68. Disponible http://www revmultimed sld.cu/index.php/mtm/article/view/200

9. Martínez Trujillo N. La formación de posgrado en Salud Pública para el ejercicio interdisciplinar en Enfermería. Educación Médica Superior [en línea]. septiembre de 2015 [citado 10 de junio de 2017];29(3):0-0. Disponible http://scielo.sld.cu/scielo.php?script=sci_abstract\&pid=S0864$21412015000300013 \&$ Ing=es\&nrm=iso\&tlng=es 